УДК 617-089

\title{
THE EFFECT OF MILD COGNITIVE IMPAIRMENT ON EEG TOPOGRAPHIC CHANGES AFTER ON-PUMP CORONARY ARTERY BYPASS GRAFTING
}

\author{
I.V. TARASOVA, O.V. MALEVA, R.S. TARASOV, O.L. BARBARASH, L.S. BARBARASH \\ Federal State Budgetary Institution Research Institute for Complex Issues of Cardiovascular Diseases, \\ Kemerovo, Russia
}

The purpose. Previously it was shown that on-pump coronary artery bypass grafting (CABG) induced cerebral ischemia and cognitive decline. The patients with mild cognitive impairment $(\mathrm{MCl})$ could be a high-risk group of CABG-associated cognitive decline. Non-invasive neuromonitoring can provide information regarding subclinical symptoms and topography of cerebral ischemia. The aim of this study was to investigate the topographic changes in electroencephalogram (EEG) spectral power in coronary artery disease (CAD) patients with or without $\mathrm{MCI}$ before and after on-pump CABG.

Methods: 62 males with CAD were divided into two groups according to their Mini-Mental State Examination: without $\mathrm{MCl}(\mathrm{n}=37)$ and with $\mathrm{MCl}(\mathrm{n}=25)$. Clinical factors were assessed, including the severity of coronary lesions (SYNTAX score), the left ventricular ejection fraction (LVEF). Eyes-closed rest EEG was recorded from 62 channels positioned according to the International 10-20 system. Spectral EEG power was calculated for frequencies from 0.1 to $50 \mathrm{~Hz}$.

Results: The patients with $\mathrm{MCl}$ had theta-1 $(4-6 \mathrm{~Hz}$ ) power increase 7-10 days after on-pump CABG only in frontal and fronto-central clusters of right and left hemispheres as compared to the preoperative values, whereas in the groups without $\mathrm{MCl}$ this effect was widespread over the brain. In the beta- 1 band $(13-20 \mathrm{~Hz}), \mathrm{CABG}$ patients with $\mathrm{MCl}$ had power increase in the frontal, fronto-central and fronto-temporal regions of the brain cortex. Similar power changes in patients without $\mathrm{MCl}$ were observed only for occipital brain clusters.

Conclusions: CAD patients with $\mathrm{MCl}$ have demonstrated EEG signs of cortical dysfunction focused on the frontal brain areas. Cortical dysfunction in this localization can be associated with the progression of cognitive deficits, causing loss social integration in CAD patients with $\mathrm{MCI}$.

Keywords: mild cognitive impairment, EEG, theta rhythm, coronary artery bypass grafting, SYNTAX score.

\section{Introduction}

Cardiovascular disease (coronary artery disease (CAD), heart failure, hypertension, cardiac arrhythmia) is commonly associated with increased risk of cognitive impairment, even in the absence of neurodegenerative disease or stroke $[1 ; 19 ; 37 ; 34]$. Cognitive impairment can be considered as an early marker for ischemic brain damage in patients with cardiovascular disease $[9 ; 11]$. Treatment options for CAD patients usually include cardiac surgery; thus, perioperative neurological events can have a dramatically detrimental effect on the duration and quality of life [22]. Despite remarkable progress in surgical, cardiopulmonary bypass and anesthetic techniques, brain damage remains an important complication of on-pump coronary artery bypass grafting $(\mathrm{CABG})$ and is associated with cerebral perfusion deterioration $[7 ; 41]$. Meanwhile, a significant number of CABG candidates are middleaged or older people, who may suffer chronic brain ischemia and mild cognitive impairment (MCI) [18; 39]. MCI is a clinical term that includes individuals with impairment in one or more cognitive domains greater than would be expected for a person's age, but who are otherwise functionally intact and capable of living independently [21]. Kline and colleagues have found that MCI subjects had greater vulnerability to cognitive impairment post surgery [21]. The authors suggested that brain atrophy associated with surgery may be more problematic for MCI subjects, due to their cumulative atrophy at the time of surgery. Furthermore, MCI subjects would presumably have less cognitive reserve and compensatory factors working to stabilize cognition [5].

Brain ischemia may be caused by on-pumpCABG and approaches to diagnose hypoxic-ischemic brain injury are complex and controversial $[3 ; 12 ; 15]$. Multi-channel computed electroencephalography (EEG) can be used to monitor brain electrical activity and thus detect signs of ischemic brain damage that can result in postoperative neurological deficit [13]. 
The noninvasiveness and simplicity of this method makes it possible to assess the dynamics of brain function recovery during the postoperative period $[13 ; 38]$. The value of non-invasive monitoring of the brain before, during and after cardiac surgery has previously been shown to provide information about the sub-clinical symptoms of cerebral ischemia [12; 35]. EEG changes can be detailed alarm criteria for classifying ischemia during carotid endarterectomy surgery [20]. It has been demonstrated that the cerebral cortical activity decreased in patients with delirium [31]. A statistically significant relation has been found between the characteristics of intraoperative EEG and cognitive deficits for 2-3 months after on-pump cardiac surgery [16].

Quantitative analysis of EEG is promising marker associated with cognitive dysfunction and may differentiate between disease stages $[2 ; 17]$. Previous studies have reported that patients with dementia demonstrate increased slow wave activity compared to healthy subjects of the same age [42]. Babiloni et al. [2] reported higher theta rhythm power in patients with vascular MCI compared to patients with neurodegenerative MCI. Several authors have found that EEG changes in Alzheimer's disease patients are diffuse and appear as increased delta and theta activity and decreased alpha activity $[4 ; 26]$. However, little is known about EEG topography in $\mathrm{MCI}$ and non-MCI patients after surgery, especially those who have undergone on-pump CABG.

The purpose of this study was to investigate the topographic changes in spectral EEG power of CAD patients with or without MCI before and after onpump CABG.

\section{Materials and Methods}

\section{Subjects}

A cohort of 62 subjects were recruited from the Cardiology Department, State Research Institute for Complex Issues of Cardiovascular Diseases, Russia, Kemerovo. All patients gave written informed consent to participate in the prospective study aimed at assessing cognitive function in CAD patients. The study design was approved by the Ethics Committee of the Institute.

Exclusion criteria were as follows: age $>70$ years, female sex, initial depressive symptoms identified by the Beck Depression Inventory (BDI-II), dementia [sum-score on the Mini-Mental State Examination (MMSE) $\leq 24]$, and the Frontal Assessment Battery
(FAB) score $\leq 11$. We also excluded subjects with a known history of rhythm disturbances, heart failure functional class IV according to the New York Heart Association (FC NYHA), concomitant diseases (chronic obstructive pulmonary disease, malignant pathology), diseases of the central nervous system, regularly using psychoactive drugs, with any episodes of a cerebrovascular accident, and/or brain injury. All 62 patients underwent multi-spiral computed tomography to detect any nervous system abnormalities.

All patients were treated with basic, symptomatic therapy according to the general principles of treatment for patients with $\mathrm{CAD}$, heart failure, and hypertension (National Guidelines, 2009, 2008): a low-salt $(<1.5 \mathrm{~g} /$ day) and low-cholesterol diet, antiplatelet medication (acetylsalicylic acid), $\beta$-blockers (bisoprolol fumarate), angiotensin-converting enzyme inhibitors (enalapril maleate), and statins (rosuvastatin).

A total of 62 male patients were divided into two groups: those with MCI according to the Petersen's criteria [30] (mean MMSE score 26.3 $\pm 0.82, \mathrm{n}=25$ ) and those without MCI (mean MMSE 28.6 \pm 0.77 , n $=37$ ). All patients underwent standardized physical, neurological, and instrumental examinations. The examiners were blind to the cognitive status of the patients. The severity of the coronary lesions was assessed using the findings of coronary angiography (Innova 3100; GE Medical Systems, Carrollton, TX, USA) and a SYNTAX calculator (http://www. syntaxscore.com/calc/start.htm). Echocardiography was performed on expert-class equipment (Vivid 7; GE Medical Systems), with estimation of the left ventricular ejection fraction (LVEF). Cognitive function was assessed using the MMSE, FAB and the Trial Making Test (TMT), Part A. Depression (BDI-II), state, and trait anxiety (Spielberger questionnaire) scores were evaluated.

All patients underwent $\mathrm{CABG}$ under normothermic cardiopulmonary bypass (CPB). Standard anesthesia and perfusion were carried out: combined endotracheal anesthesia (diprivan, fentanyl, sevoflurane). The average number of grafts placed, the mean CPB time, and aortic cross-clamp time did not differ between the two groups (Table 1). Invasive hemodynamic monitoring, carried out during the operation, indicated that there were no episodes of hypotension. Continuous real-time monitoring of the adequacy of cortical oxygenation $(\mathrm{rSO} 2)$ was performed at all stages of the surgery (INVOS 3100; Somanetics, Troy, MI, USA). Brain hypoxia was not observed. 
Table 1

Preoperative and intraoperative characteristics.

\begin{tabular}{|c|c|c|c|}
\hline Variables & $\begin{array}{l}\text { MCI group } \\
\quad(n=25)\end{array}$ & $\begin{array}{l}\text { No-MCI } \\
\text { group } \\
(\mathrm{n}=37)\end{array}$ & $\mathrm{p}$-value \\
\hline Age (years) & $56.5 \pm 5.15$ & $56.6 \pm 5.20$ & 0.76 \\
\hline $\begin{array}{l}\text { Education } \\
8-10 \text { years } \\
14-15 \text { years }\end{array}$ & $\begin{array}{l}90 \% \\
10 \%\end{array}$ & $\begin{array}{l}52 \% \\
48 \%\end{array}$ & 0.0003 \\
\hline $\begin{array}{c}\text { FC NYHA } \\
\text { I-II } \\
\text { III } \\
\end{array}$ & $\begin{array}{l}83 \% \\
17 \% \\
\end{array}$ & $\begin{array}{l}79 \% \\
21 \% \\
\end{array}$ & 0.48 \\
\hline LVEF & $53.9 \pm 10.41 \%$ & $59.1 \pm 7.22 \%$ & 0.047 \\
\hline $\begin{array}{c}\text { SYNTAX } \\
\text { score } \\
\end{array}$ & $24.8 \pm 10.03$ & $20.9 \pm 7.21$ & 0.04 \\
\hline $\begin{array}{l}\text { Diabetes } \\
\text { mellitus }\end{array}$ & $32 \%$ & $29 \%$ & 0.9 \\
\hline ICA stenosis & $29 \%$ & $26 \%$ & 0.9 \\
\hline $\begin{array}{l}\text { CPB time } \\
(\mathrm{min})\end{array}$ & $102.8 \pm 26.08$ & $96.7 \pm 27.41$ & 0.45 \\
\hline $\begin{array}{c}\text { ACC time } \\
(\mathrm{min})\end{array}$ & $67.7 \pm 20.11$ & $61.2 \pm 16.61$ & 0.25 \\
\hline $\begin{array}{c}\text { Number of } \\
\text { grafts }\end{array}$ & $2.5 \pm 0.88$ & $2.7 \pm 0.69$ & 0.62 \\
\hline
\end{tabular}

Results are given as the mean $\pm S D$ or the percent

MCI: mild cognitive impairment; CAD: coronary artery disease; FC NYHA: functional class (New York Heart Association); LVEF: left ventricular ejection fraction; ICA: internal carotid artery; $C P B$ : cardiopulmonary bypass, ACC: aortic cross clamp.

\section{EEG Recording and Processing}

High-resolution monopolar EEG recordings (62 channels, bandwidth $0.1-50.0 \mathrm{~Hz}$ ) with eyes closed were made 3-5 days before surgery and 7-10 days after GABG in patients with and without MCI. The EEG was amplified (according to the international 10-20 system) by Neuvo SynAmps2 System (Compumedics, Charlotte, NC, USA) using a modified 64-channel cap with sintered $\mathrm{Ag} /$ $\mathrm{AgCl}$ electrodes (QuikCap; Neurosoft, El Paso, TX, USA). A reference electrode was attached to the tip of the nose and a ground electrode to the center of the forehead. All electrode impedances were $<5$ $\mathrm{k} \Omega$. The EEG was sampled at a rate of $1000 \mathrm{~Hz}$ over approximately $5 \mathrm{~min}$. The data were analyzed off-line. We performed visual inspection for eye movements, electromyographic interference, and other artifacts. Artifact-free EEG fragments were divided into 2-s epochs and underwent Fourier transformation. For each subject, EEG power values were averaged within the delta $(0.1-4 \mathrm{~Hz})$, theta-1
(4-6 Hz), theta-2 (6-8 Hz), alpha-1 (8-10 Hz), alpha-2 (10-13 Hz), beta-1 (13-20 Hz), and beta$2(20-30 \mathrm{~Hz})$ bands [13]. The EEG power values of each channel for every subject in each band were obtained. The data were log-transformed to normalize the distribution.

\section{Statistical Analysis}

The software package STATISTICA 6.0 (StatSoft, Tulsa, OK, USA) was used for all analyses of the variables. Categorical clinical data were analyzed with the $\chi 2$ Pearson adjusted Yates test. Quantitative measurements were done with the Wilcoxon and Mann-Whitney tests. Statistical analyses of EEG power data were performed on five clusters in each hemisphere, such as frontal (Fp1/2, AF3/4, F1/2, $\mathrm{Fp} 3 / 4, \mathrm{Fp} 5 / 6, \mathrm{~F} 7 / 8$ ), fronto-central (FC1/2, FC3/4, $\mathrm{FC} 5 / 6, \mathrm{C} 1 / 2, \mathrm{C} 3 / 4, \mathrm{C} 5 / 6$ ), temporal (FT7/8, T7/8, TP7/8), centro-parietal (CP1/2, CP3/4, CP5/6, P1/2, $\mathrm{P} 3 / 4, \mathrm{P} 5 / 6, \mathrm{P} 7 / 8$ ) and occipital (PO3/4, $\mathrm{PO} 5 / 6$, $\mathrm{PO} 7 / 8, \mathrm{O} 1 / 2$ ) in each frequency range (see Fig. 1). The midline sites (Fpz, Fz, etc.) were excluded.

Repeated-measures analysis of variance (ANOVA) with MCI (patients with MCI or without MCI), TIME (before CABG and 7-10 days after surgery), POSITION (five clusters) and LATERALITY (left or right hemisphere) as withinsubject factors was used. Greenhouse-Geisser correction was applied to all ANOVAs. Betweengroup differences were determined by planned comparisons. The Newman-Kells post hoc tests of dynamics of EEG variables at separate clusters were conducted. $\mathrm{P}<0.05$ was considered to be statistically significant.

\section{Results}

The patients in both groups were comparable in age, severity of heart failure (FC NYHA), the frequency and severity of internal carotid artery (ICA) stenosis (Table 1). However, MCI patients had lower level of education and more severe coronary lesions by SYNTAX scores than those without MCI. Moreover, CAD patients with MCI had lower LVEF than those without MCI.

There were no life-threatening cardiac arrhythmias, acute coronary insufficiency with progression of heart failure, cerebral accidents, or transient ischemic attacks in CABG patients during the early postoperative period.

A statistically significant reduction in LVEF was found 7-10 days after CABG compared to the 
preoperative data. LVEF reduced from $53.9 \pm 10.41 \%$ to $50.2 \pm 7.38 \%(\mathrm{p}=0.01)$ in MCI patients, and from $59.1 \pm 7.22 \%$ to $52.3 \pm 6.53 \%$ in patients without $\mathrm{MCI}$ $(\mathrm{p}=0.00002)$.

The significant factors and interactions associated with MCI-related topographic changes in EEG after CABG were obtained only for theta-1 (4-6 Hz) and beta-1 $(13-20 \mathrm{~Hz})$ frequency ranges.

ANOVA of the theta-1 power showed a significant effect of the interaction of MCI $\mathrm{x}$ TIME $x$ POSITION $(F 4,240=6.67, p=0.0015)$. Post hoc analysis revealed that patients with MCI had theta-1 power increase 7-10 days after on-pump CABG only in frontal $(p=0.00002)$ and fronto-central $(p=0.01)$ clusters of the right and left hemispheres, whereas in the groups without MCI theta-1 power increase was widespread over the brain (see Fig. 2). Betweengroup differences of the dynamics of theta-1 rhythm power were established for the occipital brain areas $(\mathrm{p}=0.02)$. Patients with $\mathrm{MCI}$ before $\mathrm{CABG}$ had higher values of thetal power as compared to the groups without $\mathrm{MCI}$, but after surgery patients without MCI had higher theta-1 power in these brain regions.

In the beta- 1 frequency range a statistically significant interaction of factors MCI $\mathrm{x}$ TIME $\mathrm{x}$ POSITION (F4, $240=5.93, p=0.001)$ was also found. CABG patients with MCI had an increase in beta 1 power on the frontal $(p=0.0003)$, frontocentral $(p=0.007)$ and fronto-temporal $(p=0.001)$ regions of the brain cortex (see Fig. 3) as compared to the preoperative values. In the group without MCI similar trend was observed only in occipital brain clusters $(\mathrm{p}=0.04)$.

No between-group differences of the dynamics of beta1 rhythm power were found.

\section{Discussion}

Our study has found that CABG patients demonstrated EEG signs of brain dysfunction and damage (an increase in theta- 1 and beta- 1 powers) in the postoperative period. Golukhova et al. [13] have previously demonstrated similar results. The authors suggested intraoperative cerebral ischemia, provoked by hypoperfusion and microemboli, to be the cause of EEG changes. In the other study, Siepe et al. found that a physiological level of brain perfusion during cardiopulmonary bypass (CPB) $(80-90 \mathrm{~mm} \mathrm{Hg})$ is associated with a lower incidence of postoperative cognitive dysfunction and delirium
[33]. The failure of autoregulation may be caused not only by hypoperfusion and nonpulsatile blood flow during CPB, but also vasomotor drugs [14]. However, low cardiac output may be provoked by surgical manipulations during $\mathrm{CABG}$, leading to deterioration of the central perfusion pressure and impaired blood flow [27]. Moreover, blood-brain barrier damage, caused by CPB-induced inflammation, cannot be excluded. Both inflammation and microemboli are often considered to play a major role in postoperative brain dysfunction [32; 33].

The electrical brain function depends critically on the cerebral blood flow and oxidative metabolism of glucose, delivered to the brain tissue via the arterial blood supply. Ischemia-induced EEG alterations includes slowing (an increase of power and amplitude slow waves) during subacute and chronic ischemia [6;16;28]. Kramberger et al. [23] suggested that EEG slowing in combination with episodic EEG abnormalities is a probable marker of neurodegeneration. In our previous studies, we have reported that theta-1 power increased in CAD patients after on-pump coronary artery bypass and was more pronounced in patients with mild and moderate carotid stenoses [38]. A beta-1 power changes in CAD patients after on-pump coronary artery bypass considered as an unambiguous pathological sign of postoperative cortex dysfunction [13] and can be described in terms of ischemia-reperfusion effects on brain EEG oscillations [28].

In this study, we demonstrated that EEG signs of cortical dysfunction were global, without topographic specificity in CAD patients without MCI, whereas MCI patients had postoperative theta- 1 and beta- 1 power changes in the frontal and central areas of the cerebral cortex.

It is known that the frontal cortex regulates cognitive activities such as decision-making, response inhibition, planning and memory [10;25]. In the study of van Dam et al. [40] MCI subjects demonstrated an attention deficit with decreased neural activation in the prefrontal and anterior cingulate cortices. Other studies have shown that the frontal lobes are the most vulnerable to the damaging effects of ischemia, drugs and alcohol [8; 29]. According to our data, patients with MCI had higher SYNTAX scores. This indicator may be an indirect correlate of brain vessel lesions. It has previously been shown that the severity of carotid stenoses and the severity of CAD are significantly correlated [24; 36]. Accordingly, the cortical dysfunction on EEG 
localized in the frontal cortex can be critical, and is associated with further progression of cognitive deficits in patients with MCI.

Importantly, before surgery MCI patients had higher theta-1 power values than the non-MCI group in occipital brain areas, where alpha rhythm is typically dominant in healthy subjects. We can assume that these EEG changes reflect the subcortical activation following to chronic brain ischemia.

Our study had several limitations. First, making additional studies for assessing atherosclerotic changes in the intracranial arteries might be useful to confirm our findings concerning ischemic brain damage in CAD patients. Secondly, our sample set was not large $(n=62)$ that was not possible to show the EEG power differences between the groups with and without MCI, so further studies are required.

\section{Conclusion}

CAD patients with MCI have demonstrated EEG signs of cortical dysfunction in the form of increasing theta- 1 and beta- 1 power focused on the frontal, fronto-central and fronto-temporal brain areas, whereas in the group without MCI an increase theta-1 power was widespread over the brain in early postoperative period of CABG. The frontal cortical dysfunction can be associated with the progression of cognitive deficits, causing the loss of social integration in $\mathrm{CAD}$ patients with MCI.

\section{References}

1. Alwerdt, J., Edwards, J.D., Athilingam, P., O'Connor, M.L., Valdés, E.G. Longitudinal differences in cognitive functioning among older adults with and without heart failure. J Aging Health., 2013; 25(8): 1358-1377. doi: 10.1177/0898264313505111.

2. Babiloni C., De Pandis M.F., Vecchio F., Buffo P., Sorpresi F., Frisoni G.B. et al. Cortical sources of resting state electroencephalographic rhythms in Parkinson's disease related dementia and Alzheimer's disease. Clin Neurophysiol. 2011; 122(12): 2355-2364. doi: 10.1016/j. clinph.2011.03.029.

3. Bennys, K., Rondouin, G., Benattar, E., Gabelle, A., Touchon, J. Can event-related potential predict the progression of mild cognitive impairment? J. Clin. Neurophysiol., 2011; 28(6): 625-632. doi: 10.1097/WNP.0b013e31823cc2d3.
4. Bonanni L., Thomas A., Tiraboschi P., Perfetti B., Varanese S., Onofrj M. EEG comparisons in early Alzheimer's disease, dementia with Lewy bodies and Parkinson's disease with dementia patients with a 2-year follow-up. Brain 2008; 131: 690-705. doi: 10.1093/brain/awm322.

5. Buckner R.L. Memory and executive function in aging and $\mathrm{AD}$ : multiple factors that cause decline and reserve factors that compensate. Neuron., 2004; 44(1): 195-208.

6. Butz, M., Gross, J., Timmermann, L., Moll, M., Freund, H.J., Witte, O.W. et al. Perilesional pathological oscillatory activity in the magnetoencephalogram of patients with cortical brain lesions. Neurosci Lett., 2004; 355(1-2), 93-96.

7. Carrascal, Y., Guerrero, A.L. Neurological damage related to cardiac surgery: pathophysiology, diagnostic tools and prevention strategies. Using actual knowledge for planning the future. Neurologist., 2010; 16(3), 152-164. doi: 10.1097/ NRL.0b013e3181bd602b.

8. Crews, F.T., Boettiger, C.A. Impulsivity, frontal lobes and risk for addiction. Pharmacol Biochem Behav. 2009; 93(3): 237-247. doi: 10.1016/j.pbb.2009.04.018.

9. de la Torre, J.C. Cardiovascular risk factors promote brain hypoperfusion leading to cognitive decline and dementia. Cardiovasc Psychiatry Neurol. 2012; 2012: 367516. doi: 10.1155/2012/367516

10. de Souza L.C., Guimarães H.C., Teixeira A.L., Caramelli P., Levy R., Dubois B. et al. Frontal lobe neurology and the creative mind. Front Psychol. 2014; 5: 761.

11. Edman, A., Edenbrandt, L., FredénLindqvist, J., Nilsson, M., Wallin, A. Asymmetric cerebral blood flow in patients with mild cognitive impairment: possible relationship to further cognitive deterioration. Dement Geriatr Cogn Dis Extra, 2011; 1(1), 228-236. doi: 10.1159/000329447.

12. Gehring, H., Meyer zu Westrup, L., Boye, S., Opp, A., Hofmann, U. Transcranial doppler, EEG and SEP monitoring. Applied Cardiopulmonary Pathophysiology, 2009; 13: 26-00.

13. Golukhova, E.Z., Polunina, A.G., Lefterova, N.P., Begachev, A.V. Electroencephalography as a tool for assessment of brain ischemic alterations after open heart operations. Stroke Res Treat., 2011; 2011, 980873. doi: 10.4061/2011/980873.

14. Gottesman R.F., Hillis A.E., Grega M.A., Borowicz L.M., Selnes O.A., Baumgartner W.A. et al. Early postoperative cognitive dysfunction and 
blood pressure during coronary artery bypass graft operation. Arch Neurol., 2007; 64(8), 1111-1114.

15. Guarracino, F. Cerebral monitoring during cardiovascular surgery Curr Opin Anaesthesiol., 2008; 21(1), 50-54. doi: 10.1097/ ACO.0b013e3282f3f499.

16. Gugino L.D., Aglio L.S., Yli-Hankala A. Monitoring the electroencephalogram during bypass procedures. Semin Cardiothorac Vasc Anesth. 2004; $8(2): 61-83$.

17. Hatz, F., Benz, N., Hardmeier, M., Zimmermann, R., Rueegg, S., Schindler, C. et al. Quantitative EEG and apolipoprotein E-genotype improve classification of patients with suspected Alzheimer's disease. Clin Neurophysiol., 2013; 124(11): 2146-2152. doi: 10.1016/j. clinph.2013.04.339.

18. Hudetz, J.A., Patterson, K.M., Pagel, P.S. Comparison of pre-existing cognitive impairment, amnesic mild cognitive impairment, and multiple domain mild cognitive impairment in men scheduled for coronary artery surgery. Eur J Anaesthesiol., 2012; 29(7), 320-325. doi: 10.1097/ EJA.0b013e328354223d.

19. Huijts, M., van Oostenbrugge, R.J., Duits, A., Burkard, T., Muzzarelli, S., Maeder, M.T. et al. Cognitive impairment in heart failure: results from the Trial of Intensified versus standard Medical therapy in Elderly patients with Congestive Heart Failure (TIME-CHF) randomized trial. Eur J Heart Fail., 2013; 15(6), 699-707. doi: 10.1093/eurjhf/ hft020.

20. Isley M.R., Edmonds H.L. Jr., Stecker M. Guidelines for intraoperative neuromonitoring using raw (analog or digital waveforms) and quantitative electroencephalography: a position statement by the American Society of Neurophysiological Monitoring. J Clin Monit Comput., 2009; 23(6): 369-390. doi: 10.1007/s10877-009-9191-y.

21. Kline R.P., Pirraglia E., Cheng H., De Santi S., Li Y., Haile M., de Leon M.J., Bekker A. () Surgery and brain atrophy in cognitively normal elderly subjects and subjects diagnosed with mild cognitive impairment. Anesthesiology., 2012; 116(3): 603612. doi: 10.1097/ALN.0b013e318246ec0b.

22. Klinger, R.Y., James, O.G., Wong, T.Z., Newman, M.F., Doraiswamy, P.M., Mathew, J.P. Cortical $\beta$-amyloid levels and neurocognitive performance after cardiac surgery. BMJ Open., 2013; 3(9), e003669. doi: 10.1136/bmjopen-2013-003669.

23. Kramberger, M.G., Kareholt, I., Andersson,
T., Winblad, B., Eriksdotter, M., Jelic, V. Association between EEG abnormalities and CSF biomarkers in a memory clinic cohort. Dement. Geriatr. Cogn. Disord., 2013; 36(5-6), 319-328. Epub 2013/09/10.

24. Lee, K.B., Budoff, M.J., Zavodni, A., Polak, J.F., Jeffrey, C.J., Burke, G.L. et al. Coronary artery calcium is associated with degree of stenosis and surface irregularity of carotid artery. Atherosclerosis. 2012; 223(1): 160-165. doi:10.1016/j. atherosclerosis.2012.05.012.

25. Levy, R., Goldman-Rakic, P.S. Segregation of working memory functions within the dorsolateral prefrontal cortex. Exp Brain Res. 2000; 133(1): 23-32.

26. Moretti, D.V., Zanetti, O., Binetti, G., Frisoni, G.B. Quantitative EEG markers in mild cognitive impairment: degenerative versus vascular brain impairment. Int J Alzheimers Dis. 2012; 2012: 917537. doi: 10.1155/2012/917537.

27. Moritz, S., Rochon, J., Völkel, S., Hilker, M., Hobbhahn, J., Graf, B.M. et al. Determinants of cerebral oximetry in patients undergoing off-pump coronary artery bypass grafting: an observational study. Eur J Anaesthesiol. 2010; 27(6):542-549. doi: 10.1097/EJA.0b013e3283343ef8.

28. Moyanova, S.G., Dijkhuizen, R.M. Present status and future challenges of electroencephalography- and magnetic resonance imaging-based monitoring in preclinical models of focal cerebral ischemia. Brain Res Bull. 2014; 102: 22-36. doi: 10.1016/j.brainresbull.2014.01.003.

29. Ochfeld, E., Newhart, M., Molitoris, J., Leigh, R., Cloutman, L., Davis, C. et al. Ischemia in Broca area is associated with Broca aphasia more reliably in acute than in chronic stroke. Stroke. 2010; 41(2): 325-330. doi: 10.1161/ STROKEAHA.109.570374.

30. Petersen, R.C. Mild cognitive impairment as a diagnostic entity. J Intern Med., 2004; 256(3), 183-194.

31. Plaschke K., Fichtenkamm P., Schramm C., Hauth S., Martin E., Verch M., Karck M., Kopitz J. Early postoperative delirium after open-heart cardiac surgery is associated with decreased bispectral EEG and increased cortisol and interleukin-6. Intensive Care Med., 2010; 36(12): 2081-2089. doi: 10.1007/ s00134-010-2004-4.

32. Semmler, A., Hermann, S., Mormann, F., Weberpals, M., Paxian, S.A., Okulla, T. et al. Sepsis causes neuroinflammation and concomitant decrease of cerebral metabolism. J. Neuroinflammation. 2008; 5: 38. doi: 10.1186/1742-2094-5-38. 
33. Siepe M., Pfeiffer T., Gieringer A., Zemann S., Benk C., Schlensak C. et al. Increased systemic perfusion pressure during cardiopulmonary bypass is associated with less early postoperative cognitive dysfunction and delirium. Eur $\mathrm{J}$ Cardiothorac Surg., 2011; 40(1), 200-207. doi: 10.1016/j. ejcts.2010.11.024.

34. Silbert, B.S., Scott, D.A., Evered, L.A., Lewis, M.S., Maruff, P.T. Preexisting cognitive impairment in patients scheduled for elective coronary artery bypass graft surgery. Anesth Analg. 2007; 104(5): 1023-1028.

35. Sloan, M.A. Prevention of ischemic neurologic injury with intraoperative monitoring of selected cardiovascular and cerebrovascular procedures: Roles of electroencephalography, somatosensory evoked potentials, transcranial doppler, and near-infrared spectroscopy. Neurol Clin 2006; 24: 631-45.

36. Steinvil A., Sadeh B., Arbel Y., Justo D., Belei A., Borenstein N. et al. Prevalence and predictors of concomitant carotid and coronary artery atherosclerotic disease. J Am Coll Cardiol. 2011; 57(7): 779-783. doi: 10.1016/j.jacc.2010.09.047.

37. Stewart, R. Vascular dementia: a diagnosis running out of time. Br. J. Psychiatry. 2002; 180: 152-156.

38. Tarasova, I.V., Volf, N.V., Trubnikova, O.A.,

Для корреспонденции:

Тарасова Ирина Валерьевна

Адрес: 650002, г. Кемерово,

Сосновый бульвар, д. 6

Тел. 8(3842) 64-37-58,

E-mail: taraiv@kemcardio.ru
Barbarash, O.L. Electroencephalogram changes in patients undergoing on-pump coronary artery bypass grafting. Neurosci Behav Physiol., 2013b; 43 (5), 577-581.

39. Trubnikova, O.A., Mamontova, A.S., Syrova, I.D., Maleva, O.V., Barbarash, O.L. Does preoperative mild cognitive impairment predict postoperative cognitive dysfunction after on-pump coronary bypass surgery? J. Alzheimers Dis., 2014; 42(0), S45-51. doi: 10.3233/JAD-132540.

40. van Dam, N.T., Sano, M., Mitsis, E.M., Grossman, H.T., Gu, X., Park, Y., Hof, P.R, Fan, J. Functional neural correlates of attentional deficits in amnestic mild cognitive impairment. PLoS One. 2013; 8(1): e54035. doi: 10.1371/journal. pone. 0054035 .

41. van Harten, A.E., Scheeren, T.W., Absalom, A.R. A review of postoperative cognitive dysfunction and neuroinflammation associated with cardiac surgery and anaesthesia. Anaesthesia; 2012; 67(3): 280-293. doi: 10.1111/j.1365-2044.2011.07008.x.

42. Vecchio F., Babiloni C., Lizio R., Fallani F.V., Blinowska K., Verrienti G. et al. Resting state cortical EEG rhythms in Alzheimer's disease: toward EEG markers for clinical applications: a review. Suppl Clin Neurophysiol. 2013; 62: 223-236.

Статья поступила 06.06.2016.

For correspondence:

Tarasova Irina

Address: 6, Sosnoviy blvd., Kemerovo, 650002, Russian Federation

Tel. 8(3842) 64-37-58,

E-mail: taraiv@kemcardio.ru 